\title{
Suicide Prevention in Primary Care: Optimistic Humanism Imagined and Engineered
}

\author{
Paul R. Duberstein, $P h D^{7}$ and Anthony F. Jerant, $M D^{2}$ \\ 'Departments of Psychiatry and Family Medicine, University of Rochester Medical Center, Rochester, NY, USA; ${ }^{2}$ Department of Family and \\ Community Medicine, University of California, Davis, School of Medicine, Sacramernto, CA, USA.
}

KEY WORDS: suicide; prevention; primary care.

$\mathrm{J}$ Gen Intern Med 29(6):827-9

DOI: $10.1007 / \mathrm{s} 11606-014-2839-4$

(c) Society of General Internal Medicine 2014

A fter being mired for centuries as an object of legal debate, philosophical speculation, and religious scrutiny, suicide emerged as a pressing concern for primary care a few decades ago. Ahmedani et al. ${ }^{1}$ (this issue) remind us why primary care occupies a central spot in suicide prevention: Nearly $50 \%$ of people who died by suicide made a health care visit within 4 weeks of death. Of the $\sim 22 \%$ who saw a clinician in the week before dying, most $(\sim 75 \%)$ had no mental health diagnosis.

Ahmedani at al. conclude that there is a "dire need to improve screening" for suicide risk. Their emphasis on screening and diagnosis can be viewed as an understandable response to the once common attitude of skeptical humanism: the idea that people have a fundamental need for selfdetermination, which should not be undermined even if it leads to suicide. Skeptical humanists ${ }^{2}$ have argued that attempts to diagnose mental disorders could pathologize normative distress and devalue human uniqueness. Ahmedani et al.'s emphasis on screening and diagnosis is a product of the prevailing optimistic biomedicalism. In essence, optimistic biomedicalists believe that programmatic research on brain and cognitive processes eventually will lead nature to yield her secrets, resulting in a cure for the mental disorders that confer suicide risk. In the meantime, in the optimistic biomedicalist view, the focus of clinical efforts should be to increase the uptake of mental health services through screening. ${ }^{1}$

Skeptical humanism and optimistic biomedicalism can spawn extreme variants, with implications for patient outcomes. Skeptical humanism's perils-nihilism and abandonment - are well established. The harms of optimistic biomedicalism-medicalization, overtreatment, ignoring patient preferences, and misallocation of resources-were long overlooked, but are increasingly recognized. ${ }^{3}$ Blatant harms of optimistic biomedicalism in the care of patients atrisk for suicide (e.g., forced hospitalization) generate more fear, but the insidious harms might take a greater toll. ${ }^{4-6}$

Published online March 25, 2014
One study showed that patients are reluctant to broach the topic of depression for fear that doctors will automatically prescribe antidepressants, regardless of patient treatment preferences. ${ }^{5}$ Another showed that when clinicians are pressed for time they use screens merely to convince patients that they are depressed, not to aid diagnosis or explore symptoms. ${ }^{4}$ Biomedicalism's exuberant paternalism and skeptical humanism's nihilism could harm patients by undermining the patient-clinician relationship. Alternative care paradigms are needed. ${ }^{7}$

\section{OPTIMISTIC HUMANISM IMAGINED}

Optimistic humanism integrates therapeutic aspects of skeptical humanism and optimistic biomedicalism, while splicing off the nihilistic and exuberant extremes. Its optimism is rooted in its faith in technological and social progress. Its humanism is grounded in person-centered ${ }^{7}$ attentiveness to the fundamental psychological needs ${ }^{8}$ of all members of modern healthcare teams: patients, their families, clinicians, and ancillary staff. Cultivating an attitude of optimistic humanism could enable the identification of novel interventions not otherwise suggested by the prevailing biomedicalism. Two priorities are relevant for suicide prevention, both of which concern the patientclinician relationship:

1. Attending to clinicians' needs. An influential theory ${ }^{8}$ of motivation suggests that all people, including clinicians, have fundamental psychological needs for self-determination (autonomy, competence, relatedness). Problems arise when these needs are thwarted. It has been argued that mandates, direct to-consumer advertising, and other point-of-care intruders have adverse implications for clinician self-determination, clinician-patient communication, and patient outcomes. ${ }^{7,9}$ For example, it is plausible that routine screening for depression or suicide risk could have unexpected harms (e.g., worsening the already prevalent problem of overtreatment of people with minimal symptoms or risk) that offset the potential benefits. Ahmedani et al. call for improvements in suicide risk assessments, but appropriately caution that the United States Preventive 
Services Task Force (USPSTF) recommends depression screening only "when a system is in place to provide effective care..." Yet even under those conditions, there is a dearth of clinical trials to support a net benefit of depression screening, ${ }^{3}$ which is one reason why the UK's National Institute for Health and Clinical Excellence and the Canadian Task Force on Preventive Health Care do not recommend routine depression screening. Moreover, the USPSTF currently gives an "I" recommendation (insufficient evidence to recommend for or against) for suicide risk screening.

Like other third-party intruders, ${ }^{9}$ routinized depression and/or suicide risk screening could have the unintended consequences of thwarting clinician self-determination and alienating some patients. ${ }^{6}$ Similarly, having care managers, rather than primary care clinicians, engage patients in discussions about depression or suicide could deprive clinicians of an opportunity to leverage the therapeutic relationship ${ }^{9}$ and provide care that is responsive to patients' circumstances. ${ }^{6,10} \mathrm{~A}$ recent report in the oncology literature suggests that the patient-clinician bond might mitigate suicide risk. ${ }^{11}$ Similar studies are needed in primary care. As Julian Tudor Hart ${ }^{12}$ put it: "Life and happiness most certainly can hang on a readiness to listen, to dig beneath the presenting symptom, and to encourage a return when something appears to have been left unsaid. And not only the patient - all patients - value these things; to practice medicine without them makes a doctor despise his trade and his patients" (p. 411).

2. Attending to patients' wants. A mantra of the patient empowerment movement is that patients should get the "care they need and no less, the care they want and no more." Ahmedani et al. ${ }^{1}$ suggest that at-risk patients need to receive treatment for mental disorders, but is that their most pressing need? Many people with suicide ideation and a nontrivial proportion of people who die by suicide have no identifiable mental disorder. And what do at-risk patients want? We are aware of little systematic research on this topic. In contrast, hundreds of studies have identified numerous life circumstances that confer suicide risk, including family discord, financial difficulties, job loss, legal imbroglios, social isolation, concerns about sexual identity, and the recent diagnosis of cancer. The Interpersonal Theory ${ }^{13}$ (a prominent theory of suicide) and supporting empirical research suggest that life circumstances could generate suicidal crises by increasing experiences of burdensomeness or aloneness. Research is needed to identify the life circumstances patients want help managing, and to explore whether offering such help can reduce suicide risk and improve other patient outcomes ${ }^{10}$ while minimizing unintended consequences (e.g., increasing feelings of burdensomeness).

\section{OPTIMISTIC HUMANISM ENGINEERED}

Care delivery systems must be engineered to enable primary care providers to elicit patients' wants in a manner that allows them to provide necessary treatments while also accommodating the clinicians' own psychological needs. ${ }^{8}$ Although some primary care practices may already be designed to meet these goals, the prevailing optimistic biomedicalism has not prepared most clinicians or practices for the task. Outside the psychiatry literature, the impact of clinicians' psychological propensities on patient safety and the quality of care have received scant attention. ${ }^{14}$ The emphasis on diagnosis and treatment leaves little room for genuinely eliciting patients' wants, let alone empathically responding. Many American primary care providers are self-employed and work with five or fewer partners, making it unlikely that they alone will be able to "re-engineer" to address patients' wants that have historically resided outside their scope of practice.

Yet we live in times of rapid technological and social change. Electronic and computer technologies, including electronic health records, can be used both to catalogue decisions (diagnoses, prescriptions, etc.) and to improve decision-making processes by enabling clinicians to elicit patients' preferences. We recently showed that having patients complete a brief (median use time $5 \mathrm{~min}$ ) interactive multimedia computer program (IMCP) while waiting to see their doctor led to increased clinician inquiry about suicidal thoughts, without disturbing workflow. ${ }^{15}$ Rather than simply informing patients that they are symptomatic, the individually tailored IMCP was designed to activate patients with suggestive symptoms to consider the possibility that they might be depressed, and to motivate them to raise discussion of depression during the linked visit, thereby "opening the door" for the providers to inquire about suicide. One likely advantage of tailored IMCPs over simple "screening" is less vulnerablility to focalism bias, the diversion of patient and clinician attention from relevant contextual issues.

For too long, discussions about suicide prevention in the medical literature have largely ignored the "elephant in the room"- the size and configuration of the health care workforce. A number of policy changes (e.g., detoxification of domestic gas; modification of over-the-counter medication packaging) have been associated with reductions in suicide rates. Policy changes designed to modify the health care workforce should also be explored as a means of reducing suicide.

Ahmedani et al.'s findings suggest primary care could play a key role in suicide prevention. Given that suicide only recently emerged as a topic of interest to primary care, the absence of breakthrough interventions thus far is no cause for alarm. Clinician-focused informational interventions initially appeared promising, but are neither sustainable nor sufficiently potent. Collaborative care, involving 
the co-localization of specialty mental health providers in primary care settings, is perhaps a more encouraging option, but effects on suicide mortality have not been demonstrated. The time to experiment with novel care paradigms is long overdue. Getting patients into mental health treatment is important, but we should be mindful of the downsides of optimistic biomedicalism and the availability of alternative paradigms. Suicide has many causes, and many potential solutions.

Corresponding Author: Paul R. Duberstein, PhD; Departments of Psychiatry and Family Medicine, University of Rochester Medical Center, Box PSYCH, 300 Crittenden Boulevard, Rochester, NY 14642, USA (e-mail:Paul_duberstein@urmc.rochester.edu).

\section{REFERENCES}

1. Ahmedani BK, Simon GE, Stewart C, et al. Health care contacts in the year before suicide death. J Gen Intern Med. 2014. doi:10.1007/s11606014-2767-3.

2. Szasz T. The myth of mental illness (rev. ed.). New York: Harper \& Row; 1974.

3. Thombs BD, Coyne JC, Cuijpers $\mathbf{P}$, et al. Rethinking recommendations for screening for depression in primary care. Can Med Assoc J. $2012 ; 184(4): 413-418$.
4. Baik SY, Gonzales JJ, Bowers BJ, Anthony JS, Tidjani B, Susman JL. Reinvention of depression instruments by primary care clinicians. Ann Fam Med. 2010;8(3):224-230.

5. Bell RA, Franks P, Duberstein PR, et al. Suffering in silence: Reasons for not disclosing depression in primary care. Ann Fam Med. 2011;9(5):439-446.

6. Ganzini L, Denneson LM, Press N, et al. Trust is the basis for effective suicide risk screening and assessment in veterans. J Gen Intern Med. 2013;28(9):1215-1221.

7. Duberstein PR, Wittink M, Pigeon W. Person-centered suicide prevention in primary care. In: Sullivan GR, James L, Bongar B, eds. Handbook of suicide in veterans and military populations. New York: Oxford University Press; in press.

8. Ryan RM, Deci EL. Self-determination theory and the facilitation of intrinsic motivation, social development, and well-being. Am Psychol. 2000;55(1):68-78.

9. Branch WT Jr. Is the therapeutic nature of the patient-physician relationship being undermined? A primary care physician's perspective. Arch Intern Med. 2000;160(15):2257-2260.

10. Weiner SJ, Schwartz A, Sharma G, et al. Patient-centered decision making and health care outcomes: An observational study. Ann Intern Med. 2013;158(8):573-579.

11. Trevino KM, Abbott $\mathbf{C H}$, Fisch $\mathbf{M J}$, Friedlander RJ, Duberstein $\mathbf{P}$, Prigerson HG. Patient-oncologist alliance as protection against suicidal ideation in young adults with advanced cancer. Cancer. in press.

12. Hart JT. Inverse care law. Lancet. 1971;1(7696):405-412.

13. Van Orden KA, Witte TK, Cukrowicz KC, Braithwaite SR, Selby EA, Joiner TE Jr. The interpersonal theory of suicide. Psychol Rev. 2010;117(2):575-600.

14. Meier D, Back A, Morrison R. The inner life of physicians and care of the seriously ill. JAMA-J Am Med Assoc. 2001;286(23):3007-3014.

15. Shah R, Franks P, Jerant A, et al. The effect of targeted and tailored patient depression engagement interventions on clinician inquiry about suicidal thoughts: a randomized control trial. J Gen Intern Med. 2014 (In Press). 